\title{
High energy in-band pumped erbium doped pulse fibre laser
}

\author{
E. L. Lim, S. U. Alam, D. J. Richardson \\ Optoelectronic Research Centre, \\ University of Southampton, SO17 1BJ, United Kingdom. \\ sua@orc.soton.ac.uk
}

\begin{abstract}
We demonstrate an inband, core-pumped master oscillator power amplifier (MOPA) with a maximum pulse energy of $1.56 \mathrm{~mJ}$ at a repetition rate of $1.25 \mathrm{kHz}$, seeded by an actively Q-switched Erbium/Ytterbium-codoped fiber (EYDF) ring laser, producing 150-ns pulses at $1562.5 \mathrm{~nm}$. To maximize energy extraction whilst minimizing signal saturation effects, a $40 \mu \mathrm{m} \mathrm{Er}^{3+}$-doped larger mode area (LMA) fiber was used as the gain medium. A $1535 \mathrm{~nm}$ single mode fiber laser was used for in-band pumping of the LMA fiber. The output beam quality $\left(\mathrm{M}^{2}\right)$ was measured to be 1.6. This is to the best of our knowledge is the highest reported pulse energy for a pulse fiber laser at $1.5 \mu \mathrm{m}$ with $\mathrm{M}^{2} \sim 1.6$.
\end{abstract}

Keywords: fiber lasers, Optical amplifiers, Erbium

\section{INTRODUCTION}

Eye safe, high energy Er-based 1.55- $\mu \mathrm{m}$ pulsed source is highly desirable for application such as remote sensing and material processing, where light scattering off target can cause eye-safety concerns. Furthermore, due to the availability of the telecom grade components, the system functionality and cost of $1.55-\mu \mathrm{m}$ pulsed fiber laser is expected to be much more attractive than Tm-based $1.90-\mu \mathrm{m}$ eye-safe pulse fiber laser. Hence, $1.55-\mu \mathrm{m}$ high energy, pulse fiber laser is highly desirable for practical applications.

At present, the state-of the-art approach to generate high energy pulses is by 9xx-nm cladding pumped Erbiumdoped fiber (EDF) or Erbium/Ytterbium-codoped fiber (EYDF) lasers/amplifiers [1,2]. With EDF, a pulse energy of $\sim 17-\mathrm{mJ}\left(\mathrm{M}^{2} \sim 13-16\right)$ has been demonstrated using Erbium doped fiber (EDF) with 100- $\mu \mathrm{m}$ core diameter [1]. However, there are little published details about the experiment. With the EYDF, a maximum pulse energy of 1.15-mJ had been reported with an $\mathrm{M}^{2}$ of 1.65 and pulsewidth of $575 \mathrm{~ns}$ [2]. However, due to the uncontrolled multiple-peaks phenomenon (MPP) in the Q-switched seed source [3], the seed output pulse was temporally modulated. Thus, the initial 70-ns MPP pulse evolved into a 575-ns output pulse with even more sub-structures. This kind of pulse shape is highly undesirable for many applications.

Recently, inband-pumped Erbium doped fiber amplifiers have attracted considerable interest. For example, an optical-to-optical conversion efficiency of $\sim 80 \%$ at the "multi-watt" level has been demonstrated in a continuous-wave, core-pumped MOPA [4]. The core-pumped scheme is attractive for pulse laser systems due to the shorter pump absorption length - resulting in an inherently shorter device and reduced effective nonlinear lengths. Here, we demonstrate for the first time an all-fiber, high-energy inband, core-pumped nanosecond MOPA capable of generating pulses with energies up to $1.56 \mathrm{~mJ}$ at a repetition rate of $1.25 \mathrm{kHz}$.

\section{EXPERIMENTAL SETUP AND RESULTS}

Fig. 1 shows the experimental setup consisting of a Q-switched seed laser, a backward-cladding pumped pre-amplifier and an in-band, forward-core pumped power amplifier. The output signal from the pre-amplifier and the inband pump source at 1535-nm [4] are combined and launched into the power amplifier via a wavelength division multiplexer (WDM). 


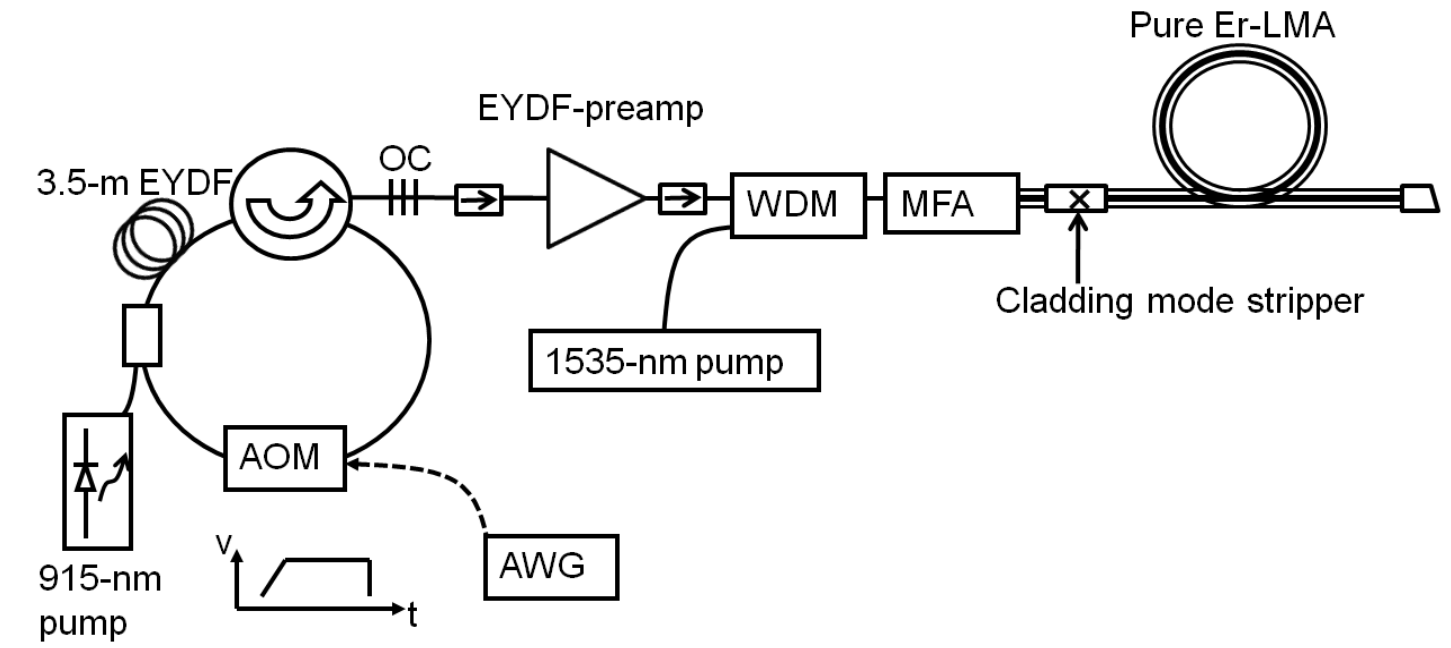

Fig. 1. Schematic view of all-fiber inband-pumped Erbium-doped pulsed fiber MOPA.

\subsection{Q-switched seed characteristics}

The seed is an acousto-optic modulator (AOM) based actively Q-switched fiber ring laser operating at a repetition rate of $1.25 \mathrm{kHz}$. The AOM was controlled by an arbitrary waveform generator (AWG) (HP 33120A). By changing the ramping rate of the trapezoidal electrical pulse, the optical rise time of the AOM can be increased to $500 \mathrm{~ns}$, such that the multi-peak pulse phenomenon (MPP) can be eliminated [3]. This generated 102-ns single-peak pulses with $13.5-\mu \mathrm{J}$ pulse energy as shown in Fig. 2.

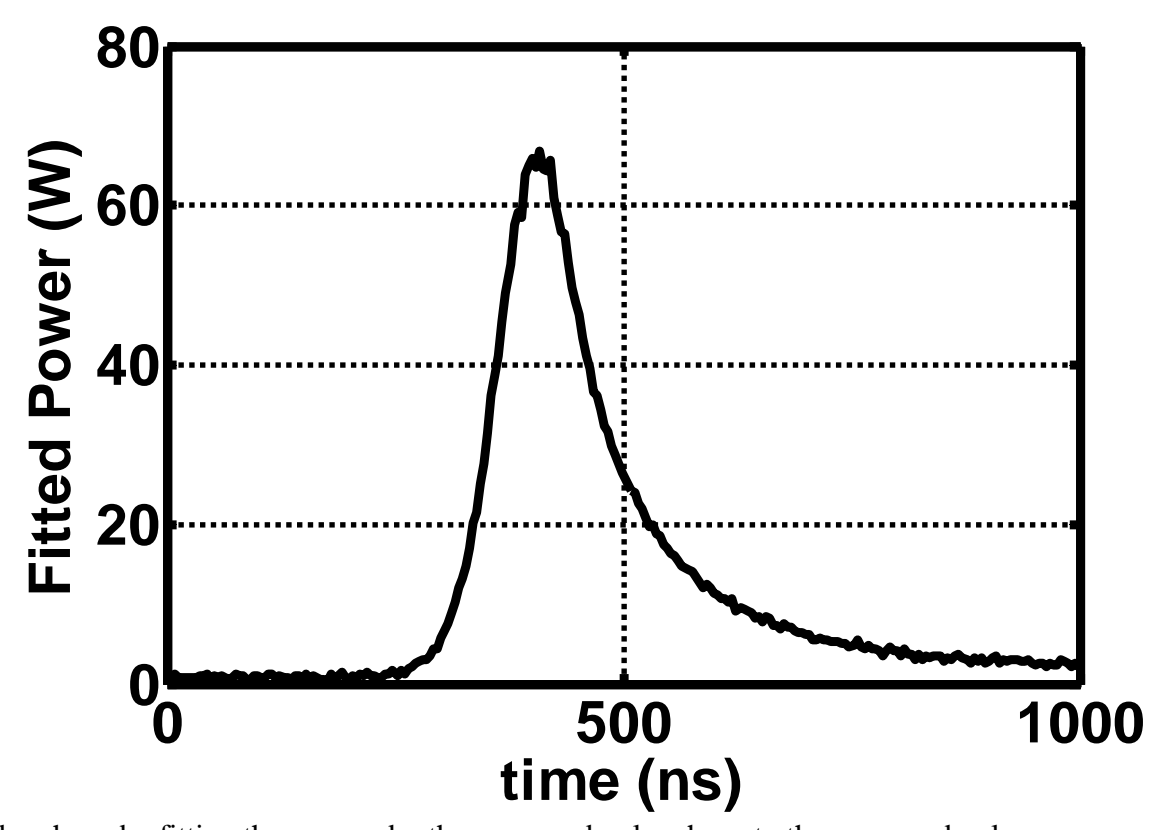

Fig. 2. The seed pulse shape by fitting the area under the measured pulse shape to the measured pulse energy.

\subsection{Pre-amplifier characteristics}

The output of the seed laser was pre-amplified by a 4.55-m long EYDF (12- $\mu \mathrm{m}$ MFD), which was backwardpumped by a 915-nm multimode laser diode using GTwave-pump technology [5]. 
As the pump power increased, the spectral components outside the central wavelength also increased due to the nonlinear spectral broadening caused by the high peak power of the pulse. These nonlinearly-broaden spectral components was filtered-out by the WDM-transmission response and resulted in the saturation of the output signal power from the WDM. Fig. 3 shows that the normalized spectrum (with respect to their respective maximum) measured directly after the WDM (solid line), before the WDM (dotted line) and the WDM-transmission response (dashed line). OSNR at the output of the WDM deteriorated due to nonlinear spectral broadening experienced by the high peak power pulse along a meter long WDM pigtail. The output pulse energy from the preamplifier into the final amplifier was chosen as $\sim 0.146-\mathrm{mJ}$. At the same time, the increase of pump power also led to an increase of the full width at half maximum (FWHM) of the pulse (as shown in Fig. 4) due to the gain saturation effect [6]. The typical fitted pulse-shapes before and after the WDM are shown in the inset. At $\sim 0.146-\mathrm{mJ}$, the measured output pulse width before the WDM was 131-ns which is $\sim 30 \%$ longer than the Q-switched seed pulse.

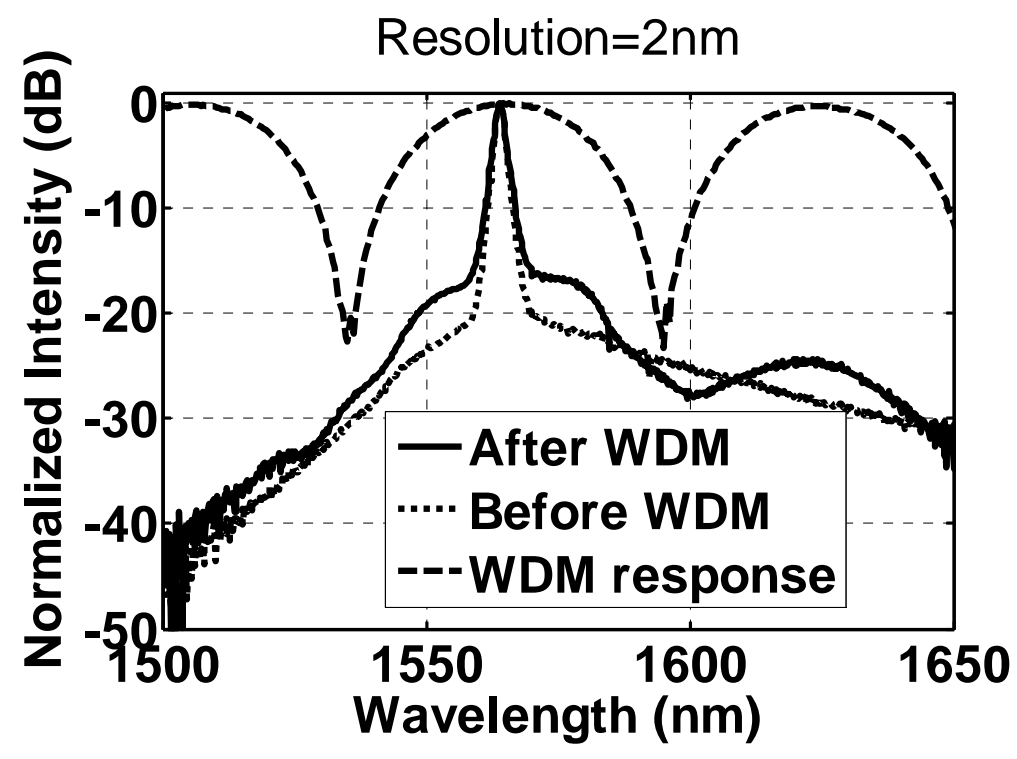

Fig. 3. The normalized output spectrum before WDM (dotted line), after WDM (solid line) and the transmission response of the WDM (dashed line).

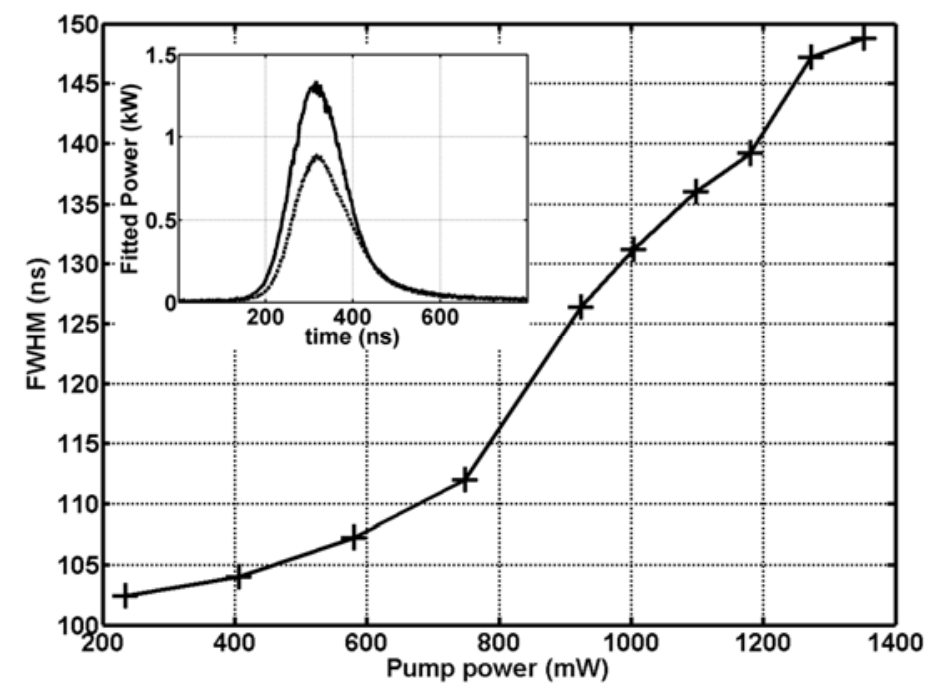

Fig. 4. The FWHM pulse width directly before the WDM. The inset shows the typical fitted pulse shape before (solid line) and after (dotted line) the WDM. 


\subsection{Final amplifier characteristics}

The power amplifier consists of a 4.9-m long, commercially available (Liekki Er 60-40/140DC) pure-Erbium doped large mode area (Er-LMA) double clad fiber with 40- $\mu \mathrm{m}$ core diameter, 0.09-NA, 140- $\mu \mathrm{m}$ cladding diameter and a cladding NA of 0.46 . The measured core absorption at $1530-\mathrm{nm}$ was $60 \mathrm{~dB} / \mathrm{m}$. To avoid facet damage due to the high pulse peak power, an end-cap was spliced to the output-end of the Er-LMA fiber. A mode field adaptor (MFA) was used in between the SMF-28 fiber (output pigtail of the WDM coupler) and the Er-LMA fiber such that the power coupling efficiency to the $\mathrm{LP}_{01}$-mode of the Er-LMA fiber can be maximized. Overall, 80\% of the pre-amplifier output power can be coupled from SMF-28 to the Er-LMA fiber core through the use of the MFA. The coupling efficiency drops down to $60 \%$ in the absence of the MFA. The maximum 1535-nm pump-power that can be coupled into the Er-LMA core was measured as $\sim 18.3 \mathrm{~W}$.

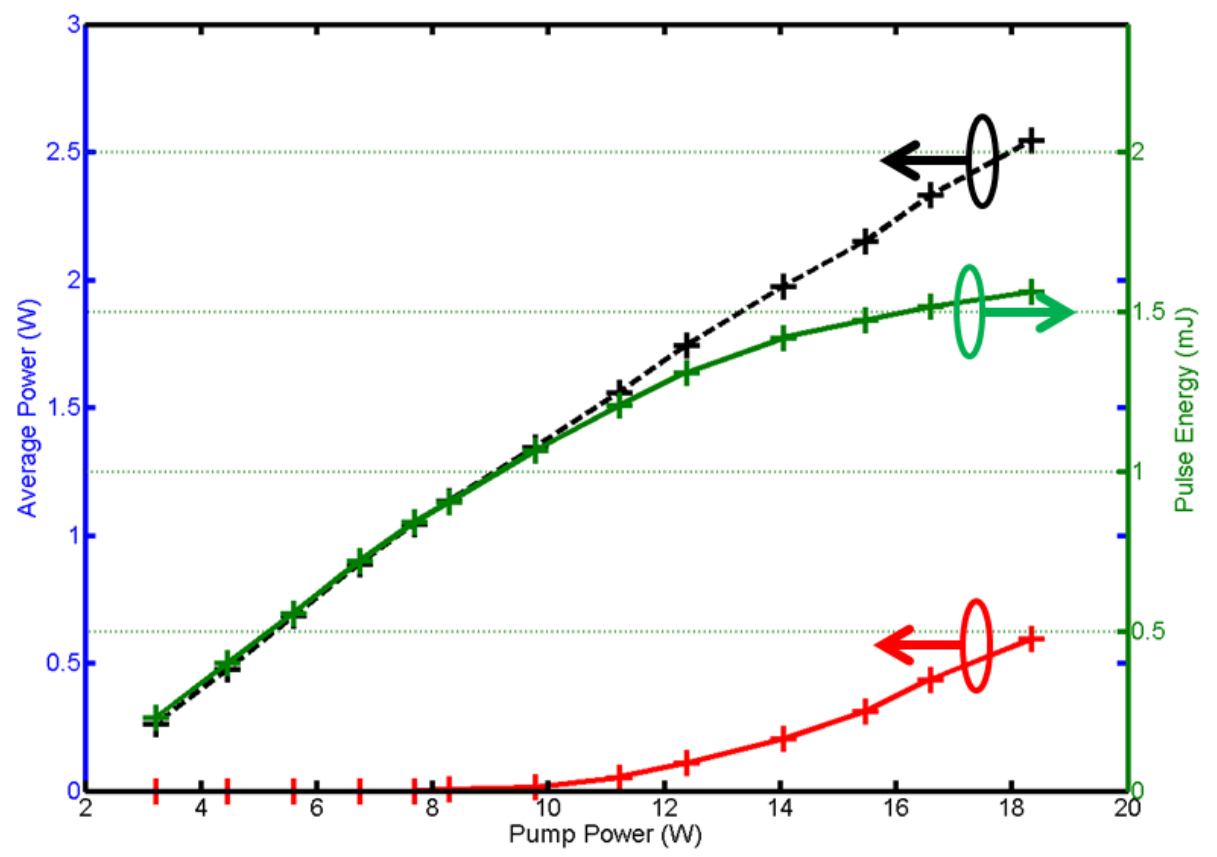

Fig. 5. The total average output power (black line), the residual pump power (red line) and pulse energy (green line) as a function of pump power.

To unambiguously discriminate the pulse power and the cw power, the pulse energy was measured directly using a high-repetition rate pyroelectric joulemeter (Gentec QE25SP-H-MT), which was insensitive to cw radiation.

The left-vertical axis of Fig. 5 shows the average power of total laser output (black line) and residual pump power (red line) vs. pump power coupled into the core of the Er-LMA. Meanwhile, the right-vertical axis shows the signal pulse energy (green line) vs. pump power. As the pump power increased, the total output power increased linearly. Up to $\sim 10$-W of pump-power, most of the total output power contained pulse signal power measured to be $\sim 1.33-\mathrm{W}$ ( 1.06-mJ pulse energy). When the pump power was further-increased, the pulse energy/signal-power started to saturate while the pump power increased rapidly. By separating the total laser output using a bulk grating, we have confirmed that the difference between the total laser output power and signal power can be accounted by the residual pump power. At the maximum available pump power, $1.95-\mathrm{W}$ of signal power was achieved corresponding to a pulse energy of $1.56-\mathrm{mJ}$. The rapid increase of residual pump power as the pulse energy saturated indicates that the saturation of output energy in our experiment was due to pump saturation.

Fig. 6 shows the measured pulse-spectrum at 3 output energy levels. At $0.55-\mathrm{mJ}$ pulse energy, there are some parasitic spectral components at the longer wavelength with $~ 8-\mathrm{dB}$ optical signal to noise ratio (OSNR). At 1.06-mJ pulse energy, the measured spectrum shows that the signal component grows faster than the parasitic spectral components at the longer wavelength. Hence, the OSNR of the spectrum has improved to $11.5 \mathrm{~dB}$. At 1.56-mJ pulse energy, the OSNR improved further to $\sim 14.6 \mathrm{~dB}$. The increase of OSNR with the pulse energy can be explained as 
follow. As the pump power increased, the inversion level also increased. This increased the energy stored in the medium and result in higher pulse energy. When the inversion level increased, the spectral position of the gain peak also shifts toward the shorter wavelength, governed by the inherent emission/absorption cross section of the Er-ions. As a result, the OSNR of the output spectrum increased.

Fig. 7 shows that the peak power of the output pulse increases from $\sim 3 \mathrm{~kW}$ to $\sim 8 \mathrm{~kW}$ as the output energy increases from $0.55 \mathrm{~mJ}$ to $1.56 \mathrm{~mJ}$. The output pulse appears to arrive earlier which can also be attributed to the gain saturation effect. At the maximum output energy of $1.56 \mathrm{~mJ}$, the pulse width was measured as $150 \mathrm{~ns}$. Finally, the $\mathrm{M}^{2}$ of the output beam was measured as $~ 1.6$ using knife edge method with energy meter as the detector.

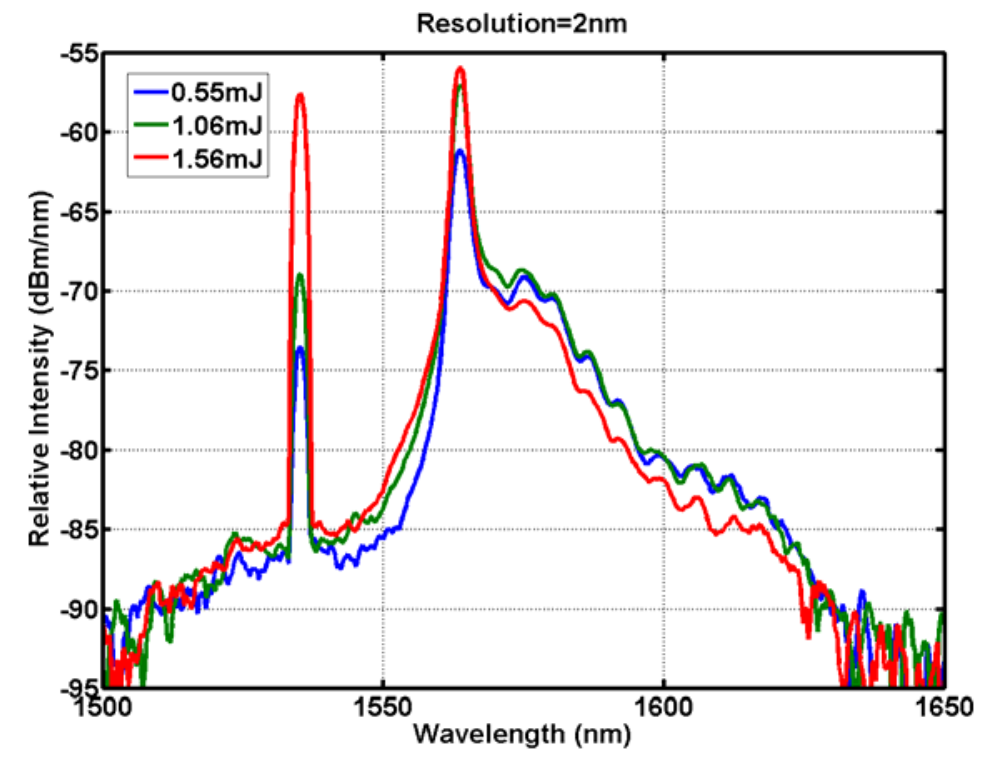

Fig. 6. The output pulse spectrum measured at 3 output energy levels.

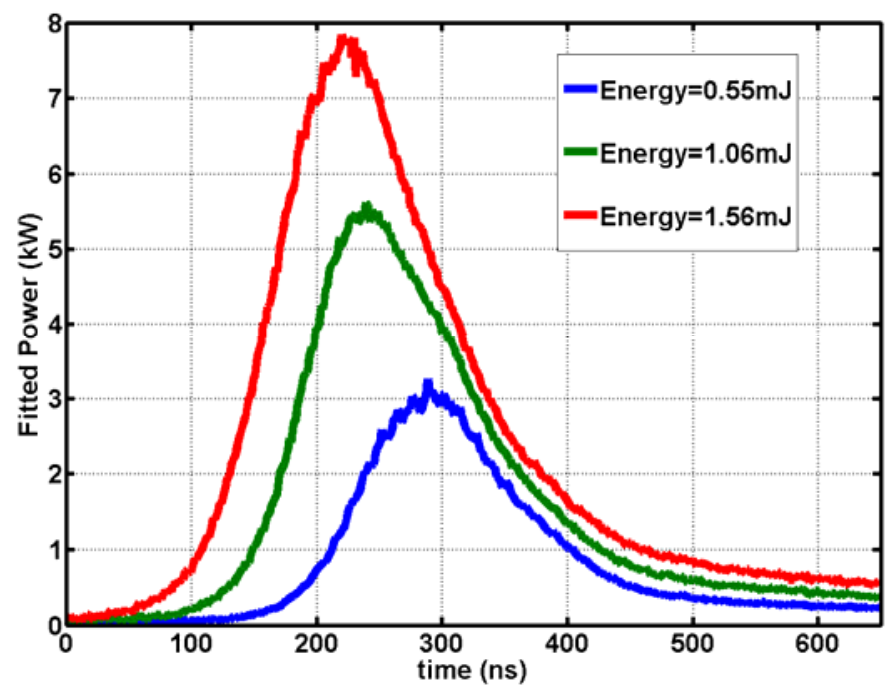

Fig. 7. The fitted output pulse shapes measured at 3 output energy levels. 


\section{CONCLUSION}

We demonstrate for the first time an all-fiber, high-energy, in-band, core-pumped nanosecond MOPA capable of generating pulses with energies up to $1.56 \mathrm{~mJ}$ at a repetition rate of $1.25 \mathrm{kHz}$. The corresponding measured pulse width was 150 -ns and the estimated pulse peak power was $\sim 8 \mathrm{~kW}$. The quality of the output beam $\left(\mathrm{M}^{2}\right)$ was measured to be $\sim 1$.6. This is to the best of our knowledge is the highest reported pulse energy for a pulse fiber laser at $1.5 \mu \mathrm{m}$ with $\mathrm{M}^{2} \sim 1$.6. Further enhancement in pulse energy was primarily limited by the saturation of the pump absorption.

\section{REFERENCES}

1. E. Lallier and D. Papillon-Ruggeri, “High energy pulsed eye-safe fiber amplifier,” Paper CJ1.5, CLEO Europe, May 22-26 May, Munich, Germany, 2011.

2. V. N. Philippov, J. K. Sahu, C. A. Codemard, W. A. Clarkson, J.-N. Jang, J. Nilsson and G. N. Pearson, “All-fiber 1.15-mJ pulsed eye-safe optical source,” Proc. SPIE 5335, 1, 2004.

3. E. L. Lim, S. U. Alam, and D. J. Richardson, "The Multipeak Phenomena and Nonlinear Effects in Q-Switched Fiber Lasers," IEEE Photonics Technology Letters, 23, 1763-1765 (2011).

4. E. L. Lim, S. U. Alam, and D. J. Richardson, "Highly efficient, high power, inband-pumped Erbium/Ytterbiumcodoped fiber laser," , CLEO , Baltimore USA, 2011.

5. A. B. Grudinin, D. N. Payne, P. W. Turner, L. J.A. Nilsson, M. N. Zervas, M. Ibsen, and M. K. Durkin, "Multi-fibre arrangements for high power fibre lasers and amplifiers," U.S. Patent 6826335, November 302004.

6. A. E. Siegman, Lasers. Mill Valley, CA.: University Science Books (1986). 